admitted to seven neonatal units in Nigeria and Kenya and diagnosed with birth asphyxia, respiratory disorders, abdominal conditions and suspected sepsis. Data was captured in REDCap** and the frequency of criteria used for each diagnosis in each NNU was analysed using SPSS.

Results 2852 neonates were included. Mean gestational age was 36 weeks (SD 4.27) and birthweight $2.42 \mathrm{~kg}$ (SD 0.94). $473(16.6 \%)$ newborns died during admission and mortality was highest amongst very-low birth weight $(<1500 \mathrm{~g})$ infants $(46.1 \%)$ and very preterm (<32 weeks' gestation) infants (41.3\%). 1230 (43.1\%) newborns were diagnosed with suspected sepsis, $874(30.6 \%)$ with respiratory conditions, 587 $(20.6 \%)$ with birth asphyxia and $71(2.5 \%)$ with abdominal conditions. For all diagnoses, the most frequently used diagnostic criteria were clinical, whilst laboratory and radiological criteria were rarely used. In addition, there was marked variation between the NNUs, including within each country, in the use of many of the criteria for each diagnosis. Using suspected neonatal sepsis as an example, temperature instability was the most used clinical criteria (1036/1230 (84.2\%) infants; varied from $72 \%$ to $93 \%$ in each $\mathrm{NNU}$ ) whereas hypotension was rarely used (1.8\% infants; $0 \%$ to $9 \%)$. For laboratory criteria, abnormal white cell count was the most commonly used $(22.7 \%$ infants; $8 \%$ to $37 \%)$ but all were used infrequently: raised C-reactive $(9.4 \%$ infants; $0 \%$ to $27 \%$ ) and presence of a pathogen in blood (16.3\% infants; $1 \%$ to $74 \%)$ and cerebrospinal fluid (1.9\% infants; $0 \%$ to $13 \%)$.

Conclusions Clinicians adopted a syndromic approach when making diagnoses but with marked variation in use of clinical criteria between NNUs. Laboratory and radiology technologies were mostly either unavailable or unaffordable. As neonatal conditions often have non-specific and overlapping clinical features, this has implications for management such as overuse of broad-spectrum antibiotics for suspected sepsis. There is an urgent need to facilitate standardisation of diagnostic pathways based on World Health Organisation and national diagnostic guidelines to optimise clinical care. In addition, investment in affordable, sustainable diagnostics suitable for low-resource settings, including point-of-care tests, is a priority. Standardisation of diagnostic pathways would also facilitate comparing disease burdens and outcomes between NNUs as a basis for research to improve neonatal outcomes.

* https://www.project-redcap.org/

$\because$ https://www.lstmed.ac.uk/nnu

\section{UNUSUAL CAUSE OF NEONATAL INTUSSUSCEPTION: THE CLUE WAS POPPING OUT OF THE EARS!}

Ahmed Kamal, Subbaraju Vatsavai, Pramod Nair. UK

\subsection{6/bmjpo-2021-RCPCH.150}

Background Infantile myofibromatosis (IM) is a rare benign tumour of infancy. Most cases of infantile myofibromatosis are believed to occur spontaneously and without a known family history of the disorder.

Objectives The objective of this case report is to highlight this rare disorder and its unusual presentation in neonatal period.

Methods A female term baby was born through an uneventful delivery with birth weight of $3.6 \mathrm{~kg}$ and did not need any neonatal resuscitation. Antenatal history was unremarkable except for high maternal BMI. There was no history of consanguinity. There was history of sarcoidosis in Dad.
Baby was noted to have bilious vomiting at 12 hours of life. On examination the observations were stable. There was no dysmorphology, but a fleshy pink peanut sized mass was noted arising from the left auditory canal completely occluding the external auditory meatus and a small soft mass on the pinna. Her abdomen was soft with no obvious distension; bowel sounds were hyperactive. The anal opening was patent. There was fresh blood in the nappy. There was minimal clear nasogastric aspirate and there were flecks of green meconium in the aspirate on gastric washout with saline. The abdominal $\mathrm{x}$ ray showed prominent gaseous bowel loops with paucity of gas in the rectum. Rest of the general and systemic examination was unremarkable. She was kept nil by mouth with nasogastric tube on free drainage and was commenced on intravenous antibiotics after screening for sepsis. The baby was transferred to the tertiary center for urgent surgical evaluation and management in view of the bilious vomiting.

Results At the tertiary center, contrast studies confirmed Malrotation. Laparotomy showed malrotation with ileocolic intussusception. Multiple nodules found in gut lining and masses noted adjacent to the ovaries bilaterally. Further imaging whole body MRI/CT chest and abdomen/pelvis/Echocardiogram showed multiple lesions involving various organs. Histopathology and immunohistochemistry confirmed myofibromatosis. Currently this child is being treated with chemotherapy.

Conclusions Our case presented with unusual findings which had a common link. Infantile myofibromas can present with solitary or multiple nodules (firm flesh coloured to pink) and are often present at birth. At first the isolated mass in the ear canal and the bilious vomiting were thought to be separate problems. However as this case was subsequently investigated we learnt that the visceral myofibromas were responsible for the intussusception.

This form of infantile myofibromatosis can cause severe, life-threatening complications depending upon the location of the lesion and the specific organs involved. MRI and ultrasounds are useful adjuncts to gain more information about the extent of these tumours however, histopathology remains the gold standard for the diagnosis of this condition. In the majority of cases, which lack visceral involvement, prognosis is excellent and spontaneous regression is often observed. On the other hand, the presence of visceral lesions is associated with a significantly poor outcomes in the absence of therapy.

\section{THE DEVELOPMENT OF A PAEDIATRIC EARLY WARNING SCORE SYSTEM (PEWS) FOR MYANMAR; THE IMPACT ON CLINICAL OBSERVATIONS AND ESCALATION OF CARE}

Le Le Nyo, Thazin Mon, Jean Bowyer, Tin Moe Phyu, Thwelt Yee Cho, Susan Rubin.

\subsection{6/bmjpo-2021-RCPCH.151}

Background The identification of the most unwell children in hospital, and the escalation of their care, is globally challenging, especially in resource-limited settings.

Objectives A Myanmar/UK working group (WG) has developed Paediatric/Neonatal Early Warning Scores and an escalation policy designed for children of all ages in Myanmar hospitals. We present here early data on the impact of a pilot project, to be followed in March 2021 by a full 4-month dataset following implementation in different settings.

Methods Review and design phase 
Since January 2020, the Myanmar Paediatric Society (MPS) and Royal College of Paediatrics and Child Health (RCPCH) have collaborated on a pilot programme to design and implement Paediatric and Neonatal Early Warning Scores for different age groups, suitable for use in all Myanmar's hospitals.

Meeting remotely and regularly, the WG reviewed existing PEWS assessment tools and escalation criteria. They agreed a standardised scoring system and escalation criteria for Myanmar and also developed a teaching package, comprising prerecorded lectures with commentaries in Burmese and supervised practice on the wards.

Pilot implementation phase Three hospitals were recruited, representing the main types of hospital in Myanmar:

- Tertiary Hospital (Medical Ward 2, Yangon Children's Hospital)

- Regional Hospital (Taunggyi Hospital)

- District Hospital (Minbu Hospital)

These hospitals audited 127 inpatient paediatric and neonatal records, assessing the completeness of observation charts and the documented response to any clinical deterioration. Following the PEWS chart implementation, the audit is ongoing.

Results Review and design phase

Initial review of existing tools from other countries showed that clinical applicability and staffing constraints in Myanmar precluded their use without some adaptation.

Clinical applicability - Myanmar hospitals treat many children with Dengue Shock Syndrome (DSS) in whom pulse pressure is an important clinical prognostic sign. The Myanmar PEWS scoring chart is the first example in global literature to incorporate this observation.

Workforce - Despite improvements, significant shortages of specialist clinicians remain and therefore escalation policies were adapted to balance the need for rapid clinical response against the backdrop of limited staffing.

Pilot implementation phase We are currently completing data collection following the implementation of the PEWS charts. Early data indicate a positive change (median 24.8\%, IQR $22.23 \%$ ) in observation chart completeness, noting that heart rate is historically well documented; the full dataset, due in early March 2021, should include over 250 charts.

Figure 1-Observation chart change from standard documentation to PEWS incorporation

Conclusions This pilot has shown that collaboration between MPS and RCPCH allowed the development of paediatric and neonatal early warning scores suitable for Myanmar hospitals with limited staffing and their clinical burden, including for the first time, Dengue Shock Syndrome. Implementing these has led to improvements in the accuracy and completeness of observations.

Further data evaluating the impact of this pilot on clinical care will be presented at the meeting.

\section{THE SAFETY AND FEASIBILITY OF DELIVERY ROOM CUDDLES DURING THE COVID-19 PANDEMIC}

Laura Crosby, Pippa Morrish, Natalia Hackett, Sarah Bates. UK

10.1136/bmjpo-2021-RCPCH.152

Background Preterm birth remains the leading cause of neonatal mortality in the UK. There are well-documented multifaceted benefits attributed to parental skin-to-skin contact with premature neonates. It is imperative that we incorporate this safely into our care for the vulnerable population of preterm infants. Delivery room cuddles (DRC) aims to optimise this at the earliest opportunity.

Objectives To evaluate implementation of routine preterm DRC at the Great Western Hospital (GWH) with particular focus on feasibility and safety. To evaluate whether practice has changed during the COVID-19 pandemic and to review areas to improve future practice.

Methods Data was collected on babies born below 35 weeks' gestation at GWH from two cohorts: those born between 1/ $11 / 2018$ and 21/12/2019, and babies born during the COVID19 pandemic (after 19/3/2020). Data was collected by retrospective review of Badger.net and of written clinical records.

The sample contained 164 neonates with a median gestation of 33 weeks and birth weight of 1849 g. Further demographics and the comparison between groups are detailed in table 1.

Results Overall, $75 \%$ of the 164 neonates had a DRC. $82 \%$ of babies were stable during the cuddle, and a further $13 \%$ clinically improved. $5 \%$ had cardiorespiratory or thermal instability. The 6 babies with instability were between 32-34 weeks gestation. 4 experienced respiratory distress during DRC prior to stabilisation. Another 32-week infant required ventilation breaths during DRC which was felt to be due to poor airway positioning. A 34-week infant had a period of bradycardia which had resolved when reassessed on the resuscitaire.

There was no significant difference in admission temperature between DRC and non-DRC groups. The percentage of

\begin{tabular}{llll} 
Abstract 274 Table 1 & & & \\
\hline & All babies & Pre-COVID & COVID \\
\hline Number & 164 & 101 & 63 \\
Median gestation (weeks) & 33 & 33 & 33 \\
Gestation range & $23-34$ & $23-34$ & $25-34$ \\
Median BW (grams) & 1849 & 1800 & 1890 \\
BW range & $549-3015$ & $549-3015$ & $870-2570$ \\
Female\% & 43 & 44 & 41 \\
Singleton\% & 70 & 63 & 79 \\
Twin\% & 27 & 30 & 21 \\
Triplet\% & 4 & 6 & 0 \\
\hline
\end{tabular}

\begin{tabular}{|c|c|c|c|c|}
\hline & & Total & Pre-COVID & COVID \\
\hline \multirow[t]{4}{*}{ Cuddle (\%) } & All babies & 75 & 70 & 84 \\
\hline & Singleton & 82 & 69 & 86 \\
\hline & Twin & 44 & 65 & 77 \\
\hline & Triplet & 100 & 100 & - \\
\hline \multicolumn{2}{|c|}{ Median cuddle length (minutes) } & 5 & 5 & 4 \\
\hline \multirow{3}{*}{$\begin{array}{l}\text { Stability during } \\
\text { cuddle (\%) }\end{array}$} & Stable & 82 & 81 & 83 \\
\hline & Improved & 13 & 14 & 11 \\
\hline & $\begin{array}{l}\text { Cardiorespiratory } \\
\text { or thermal } \\
\text { instability }\end{array}$ & 5 & 4 & 6 \\
\hline \multirow{2}{*}{$\begin{array}{l}\text { Mean admission } \\
\text { temperature }\left({ }^{\circ} \mathrm{C}\right)\end{array}$} & Cuddle & 36.8 & 36.7 & 36.8 \\
\hline & No cuddle & 36.8 & 36.8 & 36.8 \\
\hline
\end{tabular}

